\title{
A Note on the $G$-Transformation*
}

\author{
H. L. Gray** and T. A. Atchison**
}

(December 12, 1967)

Recent literature concerning the use of nonlinear transformations to evaluate numerically certain improper integrals of the first kind has shown that difficulties are encountered if the integrand $f$ is such that

$$
\lim _{t \rightarrow \infty} \frac{f(t+k)}{f(t)}=1 .
$$

This note introduces a new nonlinear transformation which is in some cases quite useful when the above limit is one. A simple example is given to illustrate the use of this transformation.

Key Words: Improper integrals, nonlinear transformations.

\section{Introduction}

In a recent paper [1], ${ }^{1}$ H. L. Gray and T. A. Atchison have introduced a nonlinear transformation for the purpose of evaluating improper integrals of the first kind. This transformation is most useful on integrals of the type

$$
\int_{a}^{\infty} f(x) d x
$$

where

$$
\lim _{t \rightarrow \infty} \frac{f(t+k)}{f(t)}=R \neq 0 \text { or } 1
$$

In this note, a new transformation is introduced which will be more suitable when $R=1$ and which reduces to the transformation defined in [1] when $R \neq 1$.

\section{The Transformation}

Let

$$
F(t)=\int_{a}^{t} f(x) d x \rightarrow S \text { as } t \rightarrow \infty
$$

When the following limit exits, let

$$
\alpha=\lim _{t \rightarrow \infty} \frac{1-R(t ; \mathrm{k})}{R(t ; k)} \frac{E(t+k)}{F(t+k)-F(t)}
$$

where $R(t ; k)=f(t+k) / f(t)$ and $E(t+k)=S-F(t+k)$.

*An invited paper.

**Present address: Texas Technological College, Department of Mathematics, P.O. Box 4319, Lubbock, Texas 79409.

${ }^{1}$ H. L. Gray and T. A. Atchison. Nonlinear transformations related to the evaluation of certain improper integrals, SIAM Journal on Numerical Analysis 4, No. 3, 363-371 (1967). 


$$
\mathrm{L}[\mathrm{F} ; \mathrm{t}, \mathrm{k}]=\mathrm{F}(\mathrm{t}+\mathrm{k})+\alpha \mathrm{R}(\mathrm{t} ; \mathrm{k}) \frac{\mathrm{F}(\mathrm{t}+\mathrm{k})-\mathrm{F}(\mathrm{t})}{\mathrm{l}-\mathrm{R}(\mathrm{t} ; \mathrm{k})},
$$

where we assume $\alpha$ exists and $\mathrm{R}(\mathrm{t} ; \mathrm{k}) \neq 1$.

In order to determine $\alpha$ from (2.2), the value of the integral $S$ appears to be necessary. The following considerations show that this is not always true. Note that

$$
\frac{1-R(t ; k)}{R(t ; k)} \frac{E(t+k)}{F(t+k)-F(t)}=\frac{E(t+k) /[1 / f(t+k)-1 / f(t)]^{-1}}{[F(t+k)-F(t)] / f(t)} .
$$

If $1 / f(t+k)-1 / f(t) \rightarrow \infty$ as $t \rightarrow \infty$, then L'Hospital's rule can be applied to the numerator and denominator separately to assist in determining $\alpha$. That is, if the limits exist and the denominator limit is not zero,

$$
\alpha=\frac{\lim _{t \rightarrow \infty}-f(t+k) / \frac{d}{d t}[1 / f(t+k)-1 / f(t)]^{-1}}{\lim _{t \rightarrow \infty}[f(t+k)-f(t)] / f^{\prime}(t)} .
$$

Note that (2.5) does not involve any integration.

THEOREM 2.1. L[F; $\mathrm{t}, \mathrm{k}] \rightarrow \mathrm{S}$ as $\mathrm{t} \rightarrow \infty$ if, and only if,

$$
\alpha \lim _{\mathrm{t} \rightarrow \infty} \mathrm{R}(\mathrm{t} ; \mathrm{k}) \frac{\mathrm{F}(\mathrm{t}+k)-\mathrm{F}(t)}{\mathrm{l}-\mathrm{R}(\mathrm{t} ; \mathrm{k})}=0 .
$$

Proof. This follows immediately from Definition 2.1.

Theorem 2.2. L[F; $\mathrm{t}, \mathrm{k}] \rightarrow \mathrm{S}$ as $\mathrm{t} \rightarrow \infty$.

Proof. If $\alpha=0$, the result is immediate from Theorem 2.1.

If $\alpha \neq 0$, then

$$
\alpha \lim _{t \rightarrow \infty} R(t ; k) \frac{F(t+k)-F(t)}{1-R(t ; k)}=\lim _{t \rightarrow \infty} E(t+k)=0
$$

and the result follows from Theorem 2.1.

The fact that $L[F ; t, k]$ converges to $S$ is of importance. However, the purpose of this transformation is to obtain a function which converges to $S$ more rapidly than the original integral. Theorem 2.3. If $\alpha \neq 0$, then $\mathrm{L}[\mathrm{F} ; \mathrm{t}, \mathrm{k}] \rightarrow \mathrm{S}$ more rapidly than $\mathrm{F}(\mathrm{t}+\mathrm{k})$ as $\mathrm{t} \rightarrow \infty$.

Proof. Since

$$
\begin{aligned}
\frac{S-L[F ; t, k]}{S-F(t+k)} & =\frac{S-F(t+k)-\alpha R(t ; k) \frac{F(t+k)-F(t)}{1-R(t ; k)}}{S-F(t+k)} \\
& =1-\alpha \frac{R(t ; k)}{1-R(t ; k)} \frac{F(t+k)-F(t)}{E(t+k)}
\end{aligned}
$$

and $\alpha \neq 0$, then

$$
\lim _{t \rightarrow \infty} \frac{S-L[F ; t, k]}{S-F(t+k)}=1-\alpha \frac{1}{\alpha}=0
$$

Note that if $\alpha=0$, then $L[F ; t, k]=F(t+k)$ and more rapid convergence is not achieved. Another interesting relation occurs if $\alpha=1$. In this case $L[F ; t, k]=G[F ; t, k]$, the nonlinear transformation introduced in [1]. The circumstances under which $L$ will reduce to $G$ are considered in the next theorem. 
Theorem 2.4. If $\lim _{t \rightarrow \infty} \mathrm{R}(\mathrm{t} ; \mathrm{k})=\mathrm{R}(\mathrm{k}) \neq 0,1$, then $\mathrm{L}[\mathrm{F}, \mathrm{t}, \mathrm{k}]=\mathrm{G}[\mathrm{F} ; \mathrm{t}, \mathrm{k}]$.

Proof. Since

$$
\begin{aligned}
\lim _{t \rightarrow \infty} \frac{E(t+k)}{F(t+k)-F(t)} & =\lim _{t \rightarrow \infty} \frac{-f(t+k)}{f(t+k)-f(t)} \\
& =\lim _{t \rightarrow \infty} \frac{R(t ; k)}{1-R(t ; k)}=\frac{R(k)}{1-R(k)},
\end{aligned}
$$

then $\alpha=1$ and the theorem follows.

The importance of the transformation $L$ lies in the fact that regardless of the limit of $R(t ; k)$, if $\alpha$ exists and is different from zero, then more rapid convergence to $S$ is still achieved. This is best illustrated by considering the following simple example in which $G[F ; t, k]$ fails to converge more rapidly than $F(t+k)$ but $L[F ; t, k]$ converges more rapidly than $G[F ; t, k]$ or $F(t+k)$.

Let $f(x)=1 /\left(1+x^{2}\right)$. Then

$$
R(t ; k)=\frac{1+t^{2}}{1+(t+k)^{2}} \rightarrow 1 \text { as } t \rightarrow \infty .
$$

However,

$$
\alpha=\lim _{t \rightarrow \infty} \frac{\left(2 k t+k^{2}\right) \int_{t+k}^{\infty} \frac{1}{1+x^{2}} d x}{\left(1+t^{2}\right) \int_{t}^{t+k} \frac{1}{1+x^{2}} d x}=\frac{2 k}{k}=2
$$

as may be determined by using eq (2.5). The transformation described in this paper becomes

$$
L[F ; t, k]=\int_{0}^{t+k} \frac{1}{1+x^{2}} d x+2 \frac{1+t^{2}}{2 k t+k^{2}} \int_{t}^{t+k} \frac{1}{1+x^{2}} d x .
$$

Taking $t=20$ and $k=0.1$,

$$
L[F ; 20,0.1] \simeq 1.571213756
$$

which is in error by about 0.0004 . It should be noted that arc $\tan 20.1$ is in error by about 0.05 while $G[F ; 20,0.1]$ is in error by approximately 0.02 .

Clearly the integral above, being very simple, could be integrated quite satisfactorily by a number of other numerical methods.-However it adequately illustrates the comparison between $L$ and $G$.

(Paper 72B1-256) 